\title{
Transformación digital: Alternativa de crecimiento para emprendedores universitarios
}

\section{Digital Transformation: Growth Alternative for University Entrepreneurs}

Christian Enrique Guerra Villalta

Universidad Politécnica Salesiana, Guayaquil, Ecuador

cguerra@ups.edu.ec

https://orcid.org/0000-0002-5379-2801

Leonor Mercedes Torres Rivadeneira

Universidad Politécnica Salesiana, Guayaquil, Ecuador

1torresr1@est.ups.edu.ec

https://orcid.org/0000-0002-1951-1973

Nicolás Armando Sumba Nacipucha

Universidad Politécnica Salesiana, Guayaquil, Ecuador

nsumba@ups.edu.ec

(iD https://orcid.org/0000-0001-7163-4252

Jorge Manuel Cueva Estrada

Universidad Politécnica Salesiana, Guayaquil, Ecuador

jcueva@ups.edu.ec

(D) https://orcid.org/0000-0002-3055-1060

Recepción: 19/04/2021 | Aceptación: 08/06/2021 | Publicación: 10/09/2021

Cómo citar (APA, séptima edición):

Guerra Villalta, C. E., Torres Rivadeneira, L. M., Sumba Nacipucha, N.A., y Cueva Estrada, J.M. (2021). Transformación digital: Alternativa de crecimiento para emprendedores universitarios. INNOVA Research Journal, 6(3), 211-226.

https://doi.org/10.33890/innova.v6.n3.2021.1744

\section{Resumen}

En un mundo en constante cambio, la transformación digital en los emprendimientos se ha vuelto una necesidad imperiosa para la sostenibilidad de los negocios, situación que debe planificarse y aplicarse en el mediano plazo. El objetivo de la presente investigación fue analizar la relación entre las variables transformación digital y emprendedores universitarios del Ecuador, para ello de forma 
exploratoria se realizó una revisión bibliográfica sobre las variables de interés, lo que permitió fundamentar el marco teórico del estudio, posteriormente y para aterrizar los conceptos al contexto ecuatoriano, se empleó el método Delphi con dos rondas de entrevistas a expertos, lo que a su vez permitió diseñar un cuestionario, el que posteriormente fue aplicado a 384 emprendedores universitarios del Ecuador, para recabar su opinión y percepción sobre la transformación digital y su efecto en la sostenibilidad de sus emprendimientos. Como principal resultado, los encuestados reconocen la importancia de la transformación digital en los emprendimientos, sin embargo, esto no se ve reflejado en las operaciones de sus negocios, lo cual puede deberse a una confusión entre la transformación digital y el solo hecho de usar herramientas tecnológicas de forma aislada en uno o más procesos. Esta ausencia de transformación digital también puede ser atribuida a la falta de: fuentes de financiamiento, conocimiento de herramientas de gestión y estudios a profundidad sobre el mercado.

Palabras claves: transformación digital; emprendimiento; tecnología; innovación; ecosistema emprendedor.

\begin{abstract}
The applied research in the course of databases in the School of Systems of a State University, using the methodology of Problem-Based Learning, had the objective of achieving competencies in the aforementioned course. It was sought to face real problems of the race. The type of study is basic, quantitative, the design is quasi-experimental, consisting of 45 students, divided into a control group and an experimental group. The test was used as a measurement instrument, which examined the solution of cases, and which were evaluated with rubrics. The dimensions of the competences were evaluated throughout the course. If we analyze the results of these dimensions: Design of the Entity Relationship Model, Design of Databases and Design of Databases from Normalization, the results are encouraging in the Post-test, since an average of 27 is evidenced in the control group, which means a moderate average in the lower limit compared to an average of 44 in the experimental group, which means an outstanding average. It is evidenced that there is a significant positive effect of Problem Based Learning in the achievement of competencies in the Database course. ( $\mathrm{sig}=0.000<0.05$ rejects the null hypothesis)
\end{abstract}

Keywords: capabilities; active learning; database; methodology; competence.

\title{
Introducción
}

La Real academia española (RAE) define al término revolución como un cambio profundo que se da normalmente en las estructuras políticas y socioeconómicas de una comunidad, varios acontecimientos hicieron que las organizaciones se redefinieran como respuesta al reto de generar resultados sostenibles y ser rentables a mediano y largo plazo. Durante los siglos XIX y XX se produjeron la primera y la segunda revolución industrial, hasta la actualidad se han identificado cuatro etapas marcadas por acontecimientos desde la inserción de cambios en ámbitos productivos hasta la transformación digital como hoy en día la conocemos.

La primera revolución industrial tuvo lugar en 1760, marcada por la construcción del ferrocarril la cual dio paso a la mecanización, se cambió la forma en que el trabajo operativo se venía realizando y mediante sistema fabril se comenzó a producir en serie. A partir de 1840 la 
segunda revolución industrial trajo consigo la tecnología; se enfocó en la mejora continua del producto a través de la innovación y reducción de costos con economías de escala (Fernández, 2016).

Hay razones para creer que las transformaciones actuales representan una prolongación de la tercera revolución industrial en el que la tecnología de la información y la electrónica se utilizaron para automatizar procesos. Sobre las bases de esta revolución se ha venido construyendo la cuarta revolución industrial a la cual conocemos como transformación digital. A diferencia de los sucesos y los tiempos que se dieron en las anteriores revoluciones, la transformación digital avanza de manera exponencial en lugar de lineal lo que conlleva la completa restructuración de los procedimientos que se vienen dando en las industrias de todos los países (Schwab, 2015).

La transformación digital es la implementación integral de las herramientas digitales a los diferentes recursos, procesos, productos, servicios y activos para mejorar la eficiencia y generar más valor para el cliente (Davenport y Westerman, 2018). El desarrollo de nuevas soluciones tecnológicas incide en varios ámbitos en donde los líderes de las organizaciones reconocen la necesidad y posibilidad de transformar verdaderamente los fundamentos de la forma en que hacen negocios, a través de la planificación continua de estrategias especializadas. A lo expuesto anteriormente puede agregarse el pensamiento de Armstrong y Kotler (2012), quienes afirman que las firmas comerciales están en la obligación de buscar continuamente nuevas formas de agregar valor a sus negocios y ofrecer valor a sus clientes, a través del uso de medios digitales situación que invita al enfoque de una transformación digital.

Los avances tecnológicos forman parte de las operaciones cotidianas de las empresas, las cuales buscan cambiar procesos mecánicos por procesos automatizados con la finalidad de ofrecer una mejor experiencia al usuario gracias a la interacción digital (Sumba, Cueva y López, 2018), esta necesidad se ve reflejada en un estudio reciente de Gartner, que prevé que la inversión en TI a escala global seguirá en alza, pasando de un crecimiento del 0,4\% en 2019 a 3,7\% hacia el 2020 y en Ecuador la inversión realizada por empresas en TIC representa 281 miles de millones (INEC, 2019).

Según el Informe Digital 2019, 13,5 millones de personas están conectadas a internet en Ecuador, y en el mismo informe en 2020 esta cifra aumentó a 13,8 millones de personas (Mentinno, 2020). De igual manera el analfabetismo digital (personas entre 15 a 49 años sin un celular activado, 12 meses sin utilización de computadora o internet) se redujo del 21,4\% en el año 2012 a 11,4\% en 2019; lo que demuestra una potencial oportunidad para emprendimientos que todavía no integran su oferta a plataformas digitales, en las cuales entre 2018 y 2019 aumentó del 2\% al $10 \%$ las transacciones en línea que no necesariamente significa una compra en línea sino que también se refiere a las operaciones realizadas a través de terminales digitales, lo cual podría constituirse en un potencial para emprendedores universitarios, considerando que estos pertenecen a las generaciones millenial y centennial, o también llamados nativos digitales.

Las condiciones de empleo juvenil en países latinoamericanos han cambiado drásticamente en las últimas décadas; mientras que entre 2000 y 2010 la proporción de jóvenes ocupados se mantuvo estable, entre 2010 y 2018 se redujo un 4\% (OIT, 2019). Según datos del ENEMDU a diciembre de 2019 en Ecuador vivían aproximadamente 3 millones de jóvenes de entre 18 a 29 
años lo que corresponde al $25 \%$ de la población en edad de trabajar y $26 \%$ de ellos se encuentran cursando niveles de educación superior. Según cifras del INEC correspondientes a diciembre de 2019, por cada 100 jóvenes de entre 18 y 29 años, 57 estaban ocupados, 6 desocupados y 37 inactivos. Sin embargo, este porcentaje de jóvenes que se encuentran ocupados no necesariamente obtienen condiciones laborales dignas, en el país la cobertura de seguridad social es reducida y centralizada en el IESS, por esta razón en 2018 la tasa de empleo adecuado entre jóvenes era de $37,7 \%$ frente al 53,8\% de aquellos que tienen un empleo inadecuado.

El término emprendimiento comienza a ganar fuerza en Ecuador desde el año 2000 (Araque, 2015). El Banco Mundial (2014) afirma, citando a Schumpeter J.A. (1911, pág. 13) que cuando el entorno es propicio, los emprendedores se arriesgan y así estimulan la productividad mediante las dinámicas de entrada y salida del mercado de las empresas, lo cual, aunado con la innovación de las ya establecidas, promueven el desarrollo económico de la sociedad. Sin embargo, a pesar de la generación de un impacto significativo estas unidades económicas tan dinámicas no garantizan la sostenibilidad en el tiempo, viéndose afectada la generación de valor y evidenciando que un alto porcentaje de ellas desaparecen en el corto plazo.

Ecuador posee una de las Tasa Emprendedora Temprana (TEA) más altas de la región; entre los cincuentas economías que se analizan en el Global Entrepreneurship Monitor(GEM) se ubicó en el puesto número 19 del ranking mundial y dentro de los ocho países que se consideran de Latinoamérica y el Caribe ocupa el segundo lugar estando por encima de países como México, Brasil o Colombia. En 2019 registro un 36.2\% superior al 2017 donde se ubicó con un 29.62\%, a pesar de esto los emprendimientos no logran crecer y tener un desarrollo sostenible, esto se evidencia en que la tasa de los negocios establecidos (es decir aquellos que superan los 42 meses) disminuyó de $15.4 \%$ en 2017 a un 14.7\% cerrando el periodo 2019 (ESPAE, 2019). Un factor principal de esta falta de sostenibilidad es la escasez de empleo, situación que obliga a los ecuatorianos a generar emprendimientos, que, en mucho de los casos son enfocados para la supervivencia y obtención de ingresos de diarios, pero carentes de planificación e innovación (Pico Versoza, 2017).

El emprendimiento es un proceso global presente en todos los países, que se ha desarrollado desde la existencia del hombre, el cual ha explorado y trabajado para procurarse siempre un sustento, y dada su relevancia, así como la del emprendedor y de las empresas en la economía actual es necesario analizar al emprendimiento bajo una lente multidimensional (Montiel y Soto, 2020). Uno de cada tres adultos se decidió a emprender en 2019 y más de la mitad de ellos son menores a 35 años y su instrucción académica es superior. De esta distribución el 10,5\% de los emprendimientos son catalogados como nuevos y un 14,9\% como establecido, de los cuales un $15,7 \%$ de sus dueños tienen estudios universitarios completos (GEM, 2019). En virtud de lo anterior, el presente trabajo se orienta a estudiar la relación entre la transformación digital y el emprendimiento, específicamente de emprendedores universitarios del Ecuador, para ello se busca responder las siguientes interrogantes: ¿Cuál es el estado actual de los emprendimientos frente a una postura de transformación digital? ¿Existe diferencia entre el uso de tecnología y la adopción de una postura de transformación digital en los emprendimientos? 


\section{Marco teórico}

\section{La transformación digital y su impacto en las organizaciones}

La evolución constante de la tecnología se refleja en la sociedad, los avances tecnológicos son equitativos y de libre acceso, y existe una disrupción en las actividades y procesos que se realizan de manera convencional; el internet y la democratización del acceso a la información hizo que las organizaciones incursionen en redes como Facebook, Twitter o Instagram para mantenerse actualizados y mantener el contacto con su audiencia. Añádase a esto, lo expuesto por Cueva, Sumba y Villacrés (2020) quienes resaltan la importancia del uso de las redes sociales, sin embargo, indican la existencia de saturación en estos medios por la gran cantidad de publicidad no enfocada a un público objetivo específico, situación que debe ser evitada.

En relación a lo anterior, conviene mencionar lo expuesto por Lalaleo, Bonilla y Robles (2021), quienes indican que las tecnologías soportadas por las telecomunicaciones han permitido que las firmas de países distantes compartan información y realicen transacciones comerciales de forma inmediata y sin intermediarios, situación que puede ser aprovechada también por pequeños emprendedores, dado que el internet les permite montar una vitrina digital que tiene la capacidad de fortalecer y maximizar la imagen institucional y el alcance de pequeños negocios. Este contexto que se ha mencionado favorece la aparición y adopción de la transformación digital.

Antes de conceptualizar el significado de transformación digital se debe hacer una diferenciación entre su similitud con el termino digitalización, con el cual es asociado erróneamente ya que este implica la incorporación de tecnología a un negocio mientras que la transformación digital involucra la automatización de procesos y la creación de fuentes de información a partir de su mejora continua, acciones que permiten que sistemas complejos sean vistos de forma más simple.

La transformación digital es un fenómeno cultural que no puede ser ignorado por las organizaciones, esta transformación va más allá de utilizar plataformas digitales, se trata de definir el negocio desde una óptica distinta y para lograrlo se requiere de cambios en la forma de pensar, gestionar y ejecutarlas. Las empresas constantemente cambian en función de las estrategias que se plantean para mantener su posición en el mercado y mejorarla, de esta manera la transformación aporta siendo un eje que modifica propuestas de valor (Giráldez, 2018).

\section{El ecosistema emprendedor en el Ecuador}

Los negocios más pequeños y en etapas temprana de desarrollo son los más vulnerables, como respuesta a las múltiples necesidades que presentaban emprendedores ecuatorianos, en 2018 la Comisión de Desarrollo Económico de la Asamblea Nacional propuso la Ley Orgánica de Emprendimiento e Innovación, la cual desde su publicación el 28 de febrero de 2020 tiene como objetivo principal establecer el marco normativo que incentive y fomente el emprendimiento, la innovación y el desarrollo tecnológico, promoviendo la cultura emprendedora e implementando nuevas modalidades societarias y de financiamiento para fortalecer el ecosistema emprendedor (LOEI, 2020). Esta nueva ley en el Ecuador servirá para fomentar el crecimiento económico del país, Moina, Morales y Córdova (2020) concluyen que existe una relación positiva entre los 
emprendedores que involucran innovación y el crecimiento económico, medido por el PIB, consolidando al emprendimiento como motor del crecimiento económico, tan necesario en países de la región latinoamericana.

Los factores de nivel de educación, genero, grado de innovación e intensión emprendedora están asociados con la autoeficacia del emprendedor; la población más común de estos estudios han sido emprendedores universitarios (Valencia y Marulanda, 2019). Según la nueva ley orgánica de emprendimiento e innovación, el ecosistema emprendedor es todo el entorno que facilita, incluye y fomenta el desarrollo de empresas y proyectos en un lugar determinado; está articulado entre actores públicos, privados, mixtos y de la economía popular y solidaria, con la academia. Según Kantis et al. (2014) para los emprendimientos dinámicos de Sudamérica esta sinergia es la clave para emprendimientos exitosos. El GEM usa variables relacionadas con la capacidad emprendedora en un país. Las cuales son: el contexto social, político y cultural, condiciones del país y su actividad emprendedora, las oportunidades y capacidades del emprendedor y finalmente la dinámica entre negocios y crecimiento (Amorós, 2011; Arafat et al., 2020).

Otro componente importante en esta investigación es la población universitaria, según la RAE la definición de universitario/a es un adjetivo para describir aquello perteneciente o relativo a la universidad; ya sea este un profesor, graduado o estudiante. Se define como educación superior a la formación académica y profesional con visión científica y académica. Dentro de la población universitaria se pueden describir dos grandes segmentos generacionales. En primer lugar, los millenials, quienes conforman aproximadamente la mitad de la fuerza laboral del mundo (United States Census Bureau, 2016) y está catalogada como la menos comprometida con su trabajo además de presentar altas expectativas de reconocimiento y comunicación (Meister y Willyerd, 2010; Adkins, 2016; Liotta, 2012).

Los millennials son una generación digital, totalmente hiperconectada, miden el éxito a través de trabajos flexibles que les permitan equilibrar trabajo y otras áreas de su vida, para mantener relaciones interpersonales sanas y estables, por esta razón un 29\% abandonaría un empleo en donde no encuentra posibilidades de desarrollo profesional y un $38 \%$ se ve emprendiendo su propio negocio en los siguientes 10 años de vida laboral (Pulgaron, 2019). La teoría de Freire (2005) sostiene que la actividad de emprender no depende de tener una estabilidad económica por parte de quien emprende, sino de la capacidad y habilidad que el individuo tenga, por esta razón lo ilustra con un triángulo invertido que se apoya sobre el emprendedor.

La generación millennial no desea tener jefes, de ninguna índole, y buscan ser agentes generadores de cambio e innovación que aporten algo significativo a la sociedad (Pontón y Márquez, 2016); pretenden trabajar en algo que realmente los apasione, por eso uno de los factores principales para emprender es la motivación.

El segundo segmento más representativo de la población universitaria son los centennials o generación $\mathrm{Z}$, nativos digitales que desean relacionarse a través de dispositivos móviles y se caracterizan por no reconocer el principio de autoridad (Espiritusanto, 2016). Para esta generación crear una empresa es sinónimo de cambio social y en general sus propuestas de negocio consisten en iniciativas para la solución de problemas de su entorno. (Ortega y Villanova, 2016). 
Las características descritas de los millenials y centennials justifican el incremento y el interés por el desarrollo de emprendimientos a nivel global y local, pues estas generaciones forman parte del dinamismo económico en la actualidad, situación que pudo ser observada en tiempos de pandemia, cuando muchos jóvenes perdieron su trabajo, pero generaron diferentes fuentes de ingreso con altos niveles de motivación, sin embargo, no puede decirse lo mismo al respecto de la innovación. En el Ecuador siete de cada diez personas que se han quedado sin empleo quieren emprender, por el nivel de escolaridad, los universitarios son los que más se arriesgan a crear nuevos negocios. Sin embargo, los emprendedores más jóvenes tienen problemas para obtener financiamiento, debido a la falta de experiencia y credibilidad (Storey, 1994; Dvoulety, Mühlböck, Warmuth y Kittel, 2018).

\section{Metodología}

El objetivo de la presente investigación consiste en analizar la relación entre las variables transformación digital y emprendedores universitarios del Ecuador como un factor que incide en el crecimiento de sus negocios, para ello se realizó un estudio de carácter exploratorio y descriptivo, integrando herramientas cuantitativas y cualitativas.

Como parte del estudio exploratorio, en primer lugar, se realizaron entrevistas a expertos en las áreas de emprendimiento sobre como la transformación digital aportaría a la sostenibilidad de los emprendimientos de los universitarios en el Ecuador. Las preguntas realizadas fueron las siguientes:

1. Según el GEM 2019, Ecuador posee la segunda TEA más alta de la región, sin embargo, también posee la tasa de salida más alta (es decir negocios que no se mantienen en el tiempo), ¿Cuáles cree son los principales motivos que generan esta situación y por qué?

2. Los indicadores demuestran que la principal motivación para emprender en nuestro país tiene que ver con la escasez de empleo, ¿qué relación tendría esta motivación de emprendimiento con la falta de sostenibilidad de negocios de jóvenes en el largo plazo?

3. Independientemente de la actividad del negocio, ¿cree que la transformación digital aportaría de igual manera o es más rentable para algún sector en específico?

4. Actualmente se destaca la infraestructura física para capacitar a jóvenes emprendedores que inician sus negocios, sin embargo, se evidencia una baja disponibilidad de capital e insuficiencia de financiamiento privado y público ¿un joven emprendedor puede encontrar en su entorno los recursos necesarios para empezar?

5. ¿Considera que la ley de emprendimiento aprobada en el Ecuador en febrero de 2020 podría generar un gran impacto en el dinamismo del ecosistema emprendedor, considerando que el país se encuentra en contracción económica y reducción del gasto público?

A través del método Delphi y utilizando como suministro los resultados de las entrevistas se diseñó y validó el cuestionario de preguntas a utilizarse para la recolección de datos, para ellos se realizaron dos rondas de validación con los expertos. 
El objeto de estudio de esta investigación son estudiantes universitarios emprendedores pertenecientes a cualquier carrera en instituciones públicas o privadas de educación superior en el Ecuador, analizando el último informe de Educación Superior, Ciencia, Tecnología e Innovación (Senescyt, 2020) se registran 60 universidades y escuelas politécnicas, de las cuales 33 son públicas y 27 privadas. Hasta el año 2018 en el Ecuador había 632.541 estudiantes universitarios matriculados. En esta misma línea, el informe del GEM 2019/2020 señala que el 36.2\% de los ecuatorianos han generado un emprendimiento (Lasio, Amaya, Zambrano y Ordeñana, 2020), considerando estas dos cifras se podría indicar que la población para el presente estudio estaría formada por 228.980 estudiantes universitarios emprendedores.

La encuesta diseñada a partir del método Delphi fue aplicada de forma online durante los meses de enero y febrero de 2021, a ecuatorianos de las tres ciudades principales del país: Guayaquil, Quito y Cuenca; que se encuentren cursando o hayan cursado una carrera universitaria en una institución de educación superior y que tengan un emprendimiento activo.

El cuestionario contiene un total de 16 preguntas cerradas, en las que se puede conocer acerca de su situación laboral, trayectoria del negocio, área a la cual pertenecen y su opinión sobre transformación digital.

Se empleó la fórmula de muestreo probabilístico para poblaciones infinitas, utilizando como nivel de confianza el $95 \%$ y un margen de error de $5 \%$.

$$
\begin{gathered}
n=\frac{Z^{2}(P)(Q)}{e^{2}} \\
\mathrm{NC}=95 \%,(\mathrm{Z}=1.96) ; \mathrm{e}=0.05 ; \mathrm{P}=0.5 ; \mathrm{Q}=0.5 \\
n=\frac{(1.96)^{2}(0.5)(0.5)}{(0.05)^{2}} \\
n=384,16 \approx 384
\end{gathered}
$$

El tamaño muestral definido fue de 384 emprendedores universitarios.

\section{Resultados}

Mediante las entrevistas realizadas se buscó analizar las experiencias de profesionales que directa o indirectamente se relacionan con el emprendimiento universitario en el Ecuador y cuyas opiniones son relevantes para alcanzar el objetivo de la investigación.

La idea más recurrente en los entrevistados fue la importancia otorgada a la generación de emprendimientos con una previa planificación y soportados por diversas tecnologías que permitan adaptarse y aprovecharse de situaciones adversas, como las ocasionadas por la pandemia del 
COVID-19, y a su vez permita plantear emprendimientos innovadores que garanticen su sostenibilidad.

Andrea Salgado, fundadora de Sailor Coffee y Brand Strategist, pondera la importancia de desarrollar y proponer un concepto diferenciador en los emprendimientos. También señala la relevancia de contar con herramientas informáticas para administrar los negocios, como pueden ser sistemas administrativos, páginas web y redes sociales, todo esto acompañado del estudio constante del mercado y creación de una identidad para la marca.

Destaca que en el país deben existir instituciones para la capacitación de jóvenes universitarios en temas legales, tributarios entre otros temas relacionados al emprendimiento, que les permitan dar estructura a los pequeños negocios desde el inicio para que se conviertan en organizaciones sólidas y conozcan acerca de los beneficios que la ley les pueda brindar.

Por su parte, Victoria Izquieta, Directora del Ecosistema de Emprendimiento en la Universidad Politécnica Salesiana sede Guayaquil, desde su experiencia sostiene que el nivel de deserción evidenciado en la tasa de salida del GEM 2019 es causado por una pobre administración del negocio, muchos estudiantes por la escasez de empleo se dejan llevar de sus emociones y por impulso comienzan a emprender sin previamente haber estudiado el entorno y operación del negocio; lo cual incide negativamente en su sostenibilidad. Añade que una de las estrategias más utilizadas por los jóvenes emprendedores es la propuesta de precios bajos y no analizan la generación de un valor diferenciador, lo cual le permita aumentar sus márgenes de ganancia.

Para Carolina Lucín, docente en Universidad Católica Santiago de Guayaquil y Universidad del Rio, uno de los factores que incide en la falta de sostenibilidad de los emprendimientos es el enfocarse solo en el producto y no en el proceso administrativo, donde no se toman en cuenta componentes como la inversión, estudio de proveedores, factor de innovación, análisis que debería proyectarse a mediano y a largo plazo. Agrega que la transformación digital aporta de manera positiva y que actualmente hay modelos de negocios que incluso lo deben ver como algo obligatorio, pero que se lo puede clasificar en niveles porque habrá organizaciones que lo necesiten más que otras dado que la implementación de estas herramientas conlleva también inversión. Actualmente es el momento propicio para que estas estrategias sean aplicadas según la magnitud y recursos que el emprendedor disponga.

Edgar Camino, docente en Universidad Laica Vicente Rocafuerte, señala que entre las principales causas de que los negocios no sobrepasen este umbral de 42 meses es la falta de financiamiento por requisitos que los incipientes emprendedores no pueden cumplir, y agrega que la academia no ha adaptado su pensum para que los potenciales emprendedores conozcan acerca de nuevas herramientas y mecanismos de financiamiento.

Finalmente, Pedro Vargas, docente de la Universidad de Especialidades Espíritu Santo y Fundador de Spidy, concuerda con los otros expertos indicando que un emprendimiento no es sostenible debido a que el emprendedor no tiene el suficiente conocimiento sobre el negocio que desea desarrollar, en su mayoría solo ha realizado una investigación poco profunda y en mucho de los casos subjetiva, previo a empezar la operación de sus negocios. Por otra parte, sostiene que otro error recurrente es la escasa inversión en promoción y publicidad del emprendimiento. 
Al respecto de la transformación digital considera que es vital en la actualidad, y que en cinco años la tecnología habrá crecido exponencialmente; el tema de seguimiento de clientes, proveedores y comunicación se debería tener un enfoque completamente digital y automatizado, e invita a la reflexión sobre la importancia de la data para ayudar a detectar problemas y descubrir oportunidades en cualquier sector o emprendimiento por más pequeño que sea.

Por otra parte, con la encuesta aplicada de forma online se recolectaron un total de 578 respuestas, pertenecientes a emprendedores universitarios, superando el tamaño muestral (384) definido en la sección de metodología y reduciendo el margen de error a 4.1\%. A continuación, en la Tabla 1, se presenta los descriptivos más relevantes de la muestra.

\section{Tabla 1}

Variables demográficas

\begin{tabular}{ccc}
\hline $\begin{array}{c}\text { Variables } \\
\text { demográficas }\end{array}$ & Frecuencia & Porcentaje \% \\
\hline \multicolumn{3}{c}{ Genero } \\
\hline Masculino & 193 & 33,9 \\
Femenino & 376 & 66,1 \\
\hline Nivel cursado a nivel universitario \\
\hline Pre universitario & 11 & 1,9 \\
1-2 semestre & 16 & 2,8 \\
3-4 semestre & 65 & 11,2 \\
5-6 semestre & 159 & 27,5 \\
7-9 semestre & 81 & 14 \\
Egresado/Profesional & 246 & 42,6 \\
\hline \multicolumn{3}{c}{ Situación laboral actual } \\
\hline Dependiente & 122 & 21,1 \\
Dependiente con & 162 & 28 \\
negocio & 223 & 38,6 \\
Independiente & 71 & 12,3 \\
Desempleado &
\end{tabular}

Desarrollando las preguntas contenidas en el cuestionario, al consultarles a los encuestados, ¿Cuánto tiempo tiene funcionando su negocio? Tomando como escala las definiciones GEM en donde se clasifica a los emprendimientos por: potenciales (planean iniciar actividades en los próximos 6 meses), nacientes (menos de 3 meses), nuevos (más de 3 meses y menos de 42meses) y establecidos (más de 42 meses). Los resultados determinan que cerca del 50\% tienen un emprendimiento considerado como nuevo siendo la ponderación más alta, seguido de aquellos con un negocio naciente. 


\section{Figura 1}

\section{Clasificación de emprendimientos y su tiempo funcionando}

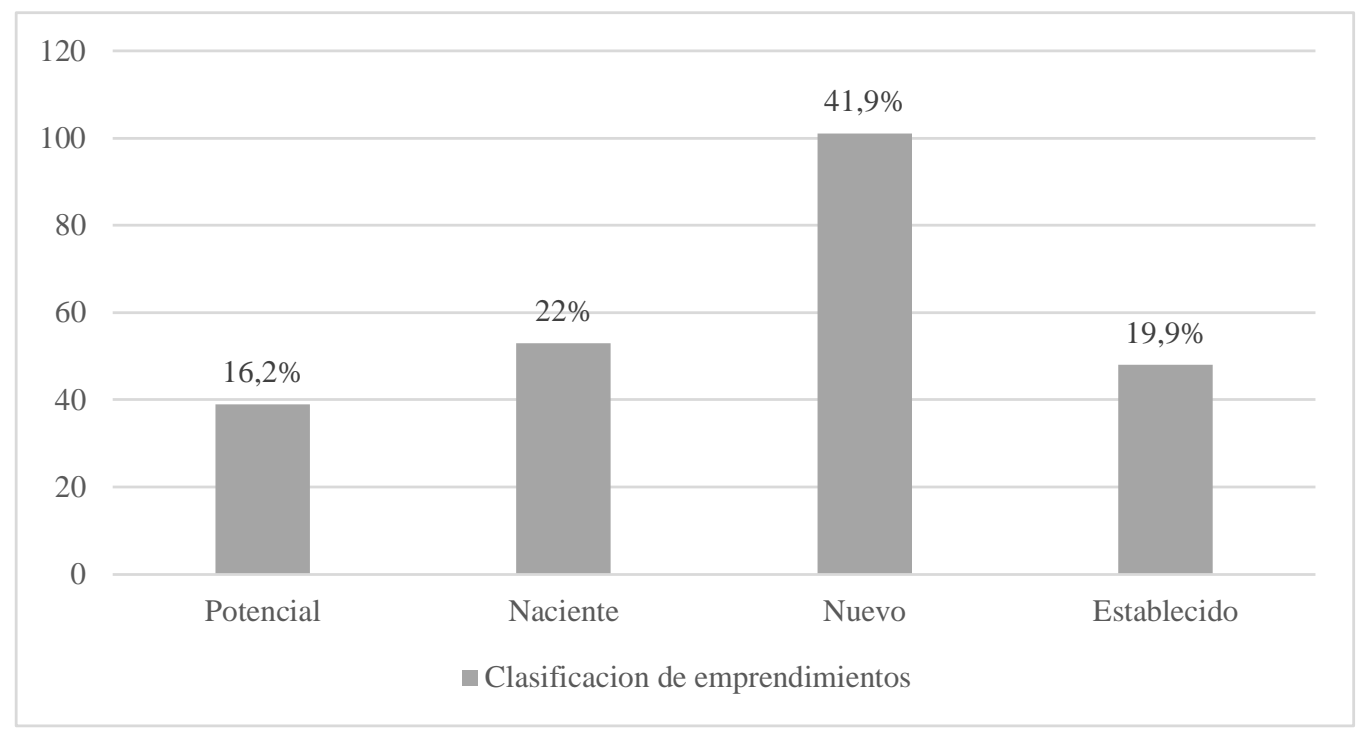

En la siguiente pregunta se indaga acerca de la actividad económica del negocio, las respuestas se concentraron en la categoría de repostería, alimentos, bebidas alcohólicas, seguida de la venta al por menor/mayor de ropa, calzado y accesorios junto a regalos y manualidades, entre estas dos últimas la variación porcentual fue mínima. Dado que actualmente existen una gran variedad de emprendimientos, los encuestados pudieron agregar la actividad de su negocio en caso de no encontrarse entre las opciones predeterminadas, las que se repitieron más fueron: prestación de servicios profesionales (contabilidad, marketing, entre otros) y asesoría, educación, venta de productos tecnológicos.

Acerca de la situación laboral de los encuestados estaban las opciones: desempleado, dependiente, dependiente con negocio e independiente. Predominaron y se tomó en consideración las respuestas dadas por aquellos que escogieron independiente o dependiente con negocio, puesto que, es de interés para la investigación conocer las opiniones de este segmento de la población.

El GEM menciona cinco como las principales razones para emprender en Latinoamérica y en su último estudio ubica con mayor ponderación la escasez de empleo como la principal motivación, sin embargo, cerca del 50\% de los encuestados respondió que su principal motivación seria contar con una fuente de ingresos adicional tomando en consideración que cerca de 100 personas indicaron ser dependientes con negocio y la segunda principal motivación seria la independencia económica.

El principal problema para emprender es la falta de recursos económicos, siendo la opción más seleccionada aproximándose al 50\%, esto como efecto de que la principal fuente de capital semilla para los emprendedores son ahorros personales, al no contar con otras fuentes de financiamiento para el inicio de sus operaciones muchos arriesgan su capital. Las principales fuentes de financiamiento de los emprendedores ecuatorianos se presentan en la figura 2. 


\section{Figura 2}

Principales fuentes de capital para emprendedores en Ecuador

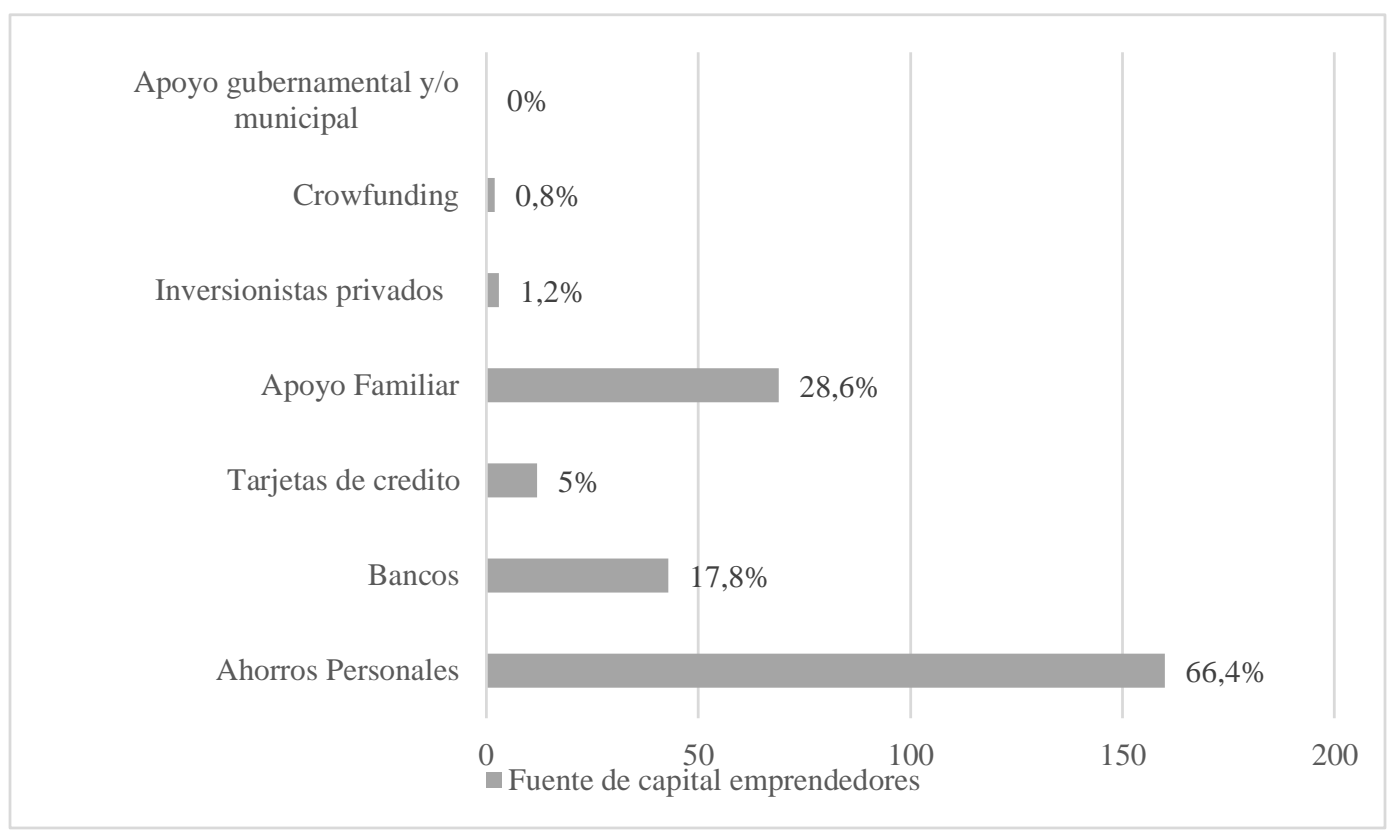

Según Decreto Ejecutivo 1113 emitido a partir de la aprobación de la Ley Orgánica de Emprendimiento se crea y actualiza el Registro Nacional del Emprendimiento (RNE), el cual brinda mejores apoyos institucionales, accesos a servicios preferenciales, como la verificación y validación de proyectos que se postulen a fondos de riesgos y acceso a descuentos con respecto a permisos de funcionamiento, notificaciones sanitarias, certificaciones de buenas prácticas de manufactura, en los servicios que ofrece el Servicio Nacional de Derechos Intelectuales (SENADI), entre otros. Sin embargo, la realidad evidenciada es que más del $90 \%$ de los encuestados no mantienen sus negocios registrados en el RNE y apenas el 0,8\% están inscritos en la Superintendencia de Compañías.

Finalmente, cerca del $50 \%$ de los emprendedores indicaron que sus ventas online generan entre el $31 \%$ al $60 \%$ de sus ingresos totales mensuales, esto puede sustentarse en las respuestas positivas sobre la percepción de la incidencia de la tecnología en sus negocios y como la transformación digital puede mejorar las operaciones de estos. Este resultado se puede observar en la figura 3. 
Figura 3

Percepción de incidencia positiva de la transformación digital en los emprendimientos

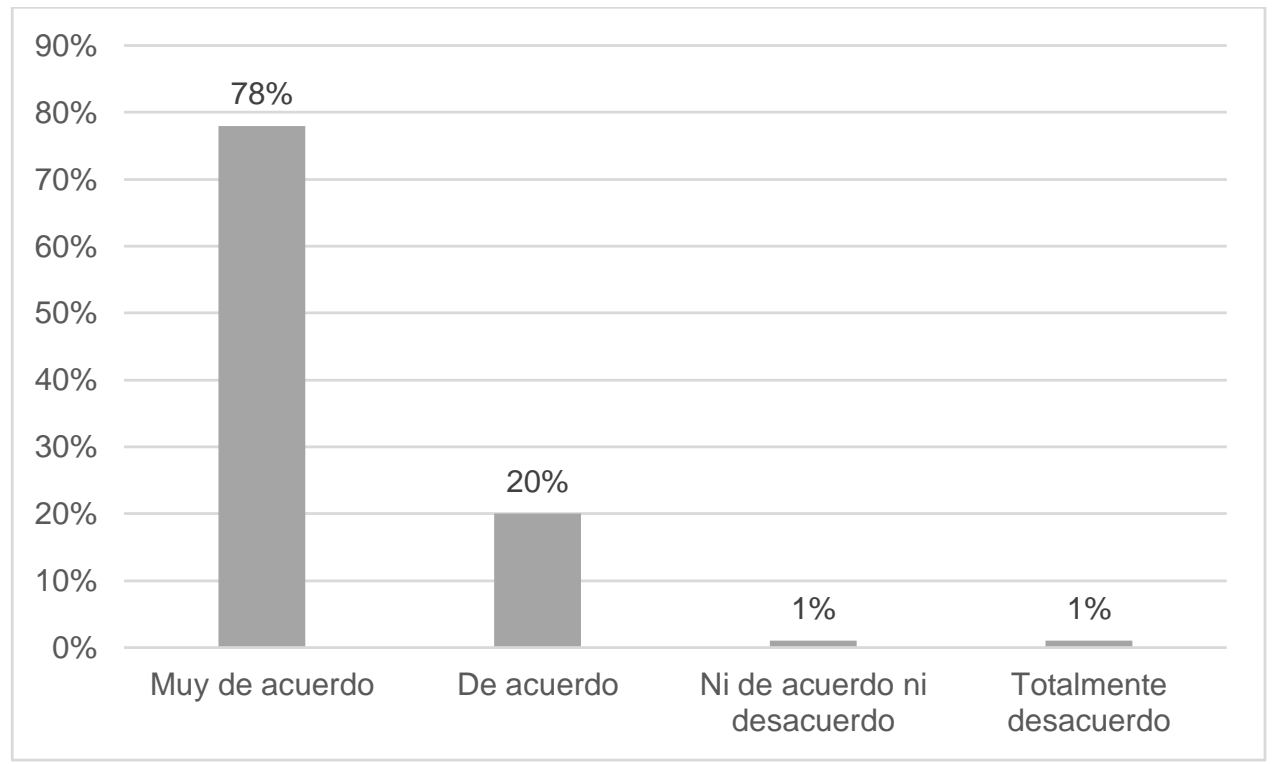

\section{Conclusiones}

Muchos medios mencionan y destacan al Ecuador como uno de los más emprendedores de Latinoamérica, de hecho, el último estudio del GEM lo ubicó en el segundo lugar, sin embargo, las oportunidades de sostenibilidad que brinda el entorno a quienes deciden seguir este camino parecieran escasas. El índice de contexto de emprendimiento nacional (NECI) presenta al Ecuador ocupando el puesto número 34 entre 54 países, situación que demuestra que el contexto ecuatoriano es medianamente favorable para empezar un negocio.

Los emprendedores deben entender que la transformación digital no significa solo la adquisición de tecnología, sino una estrategia para reinventar el modelo de negocio de la organización, situación que puede dar paso a la creación de una ventaja competitiva que le permita a la organización sostenibilidad y crecimiento en el mercado. Esta apuesta no es sencilla, debido a que los ecuatorianos utilizan mayoritariamente capital propio para iniciar sus emprendimientos, en este sentido los emprendedores deben ubicar dentro de su planificación y proyección, un conjunto de actividades y metas que les permita alcanzar paulatinamente y en un mediano plazo la transformación digital de su negocio.

Para ello, la transformación digital debe incluir: 1) la adopción de pensamiento digital en el emprendedor, 2) la gestión disruptiva en los procesos del negocio que contribuya a la mejora en la interacción con clientes y proveedores, y 3) transformación basada en datos que inicie desde generación de data que posteriormente debe ser empleada para realizar un análisis objetivo del desempeño del negocio y que permita el planteamiento de estrategias para una mejora continua y la búsqueda constante de nuevos proyectos innovadores de inversión. 
También es necesario que el ecosistema emprendedor del Ecuador se alinee con las necesidades de los emprendedores, en este sentido los centros de educación superior están obligados a transformar sus mallas curriculares, de tal forma que le brinde a la juventud emprendedora, herramientas y conocimientos pertinentes para conseguir la sostenibilidad de los nuevos negocios. En relación con esto, los emprendedores deben conocer mecanismos alternativos de financiamiento, como el crowfunding, herramienta no tradicional que permite recaudar fondos monetarios para proyectos e iniciativas.

Sobre los emprendimientos que emplean herramientas digitales, se puede señalar que el $50 \%$ de la muestra afirma usar las redes sociales como medio para potenciar su negocio, con este resultado los autores plantean las siguientes preguntas: ¿Es esto una transformación digital?, ¿es suficiente las redes sociales para construir la identidad de marca? Las redes sociales son ampliamente consumidas por la sociedad en la actualidad y sirven como medio para realizar diversas transacciones comerciales, sin embargo, se debe destacar que los negocios que además poseen páginas web oficiales brindan mayor seguridad y confianza al cliente, puesto que se presentan ante la sociedad como una organización establecida, estructurada y sólida. En esta misma línea, los emprendedores deben reemplazar completamente el uso de correos personales por cuentas de correo con dominio propio, para el desarrollo de transacciones comerciales en sus emprendimientos.

Los emprendedores son conscientes de la importancia del uso de la tecnología y de la transformación digital en sus emprendimientos, sin embargo este pensar no se observa aplicado en sus negocios, puesto que el $61 \%$ de los emprendedores encuestados indicó utilizar como forma de pago el dinero en efectivo y transferencias bancarias, que pueden considerarse como medios de pago tradicionales, dejando de lado otras opciones de pago, como tarjeta de crédito, paypal, dinero electrónico; esto evidencia que no han diseñado e implementado sitios web como medios para realizar un auténtico comercio electrónico, y que además ejerzan como sucursal virtual del negocio las 24 horas del día y colaboren en su sostenibilidad.

\section{Referencias bibliográficas}

Amorós, J. E. (2011). El proyecto Global Entrepreneurship Monitor (GEM): una aproximación desde el contexto latinoamericano. Academia. Revista Latinoamericana de Administración, (46), 1-15

Arafat, M. Y., Saleem, I., Dwivedi, A. K. y Khan, A. (2020). Determinants of agricultural entrepreneurship: A GEM databased study. International Entrepreneurship and Management Journal, 16(1), 345-370. https://doi.org/10.1007/s11365-018-0540-5

Asamblea Nacional República del Ecuador (2020). Ley Orgánica de Emprendimiento e Innovación. Quito: Asamblea Nacional República del Ecuador

ASAMBLEA NACIONAL. (2020, 18 febrero). Ley Orgánica de Emprendimiento e Innovación https://www.gob.ec/regulaciones/ley-organica-emprendimiento-innovacion 
Cueva-Estrada, J., Sumba-Nacipucha, N., Villacrés-Beltrán, F. (2020). El marketing de influencias y su efecto en la conducta de compra del consumidor millenial. Suma de Negocios, 11(25), pp. 99-107. http://doi.org/10.14349/sumneg/2020.V11.N25.A1

Dvouletý, O., Mühlböck, M., Warmuth, J. y Kittel, B. (2018). 'Scarred' young entrepreneurs. Exploring young adults' transition from former unemployment to self-employment. $\begin{array}{llll}\text { Journal of } \quad \text { Youth } & \text { Studies, } & \text { 21(9), }\end{array}$ https://doi.org/10.1080/13676261.2018.1450971

ESPAE. (2020, 17 julio). Global Entrepreneurship 2019. ESPAE Escuela de Negocios. https://www.espae.espol.edu.ec/gem/

ESPAE. (febrero, 2020). Empleo, subempleo y desempleo juvenil: El caso de los jóvenes de Posorja. https://bit.ly/3gLwah5

Espiritusanto, Ó. (2016). Generación Z: Móviles, redes y contenido generado por el usuario. En Revista de estudios juventud, 111-126

Freire, A. (2005). Pasión por emprender: De la idea a la cruda realidad. Colombia Norma

Kantis, H., Federico, J. y Trajtenberg, L. (2014). Middle-class entrepreneurs and their firms: A regional view and international comparison. En E. Lora y F. Castellani (Eds.), Entrepreneurship in Latin America. A step up the social ladder (pp. 53-80). Washington: Banco Mundial. https://doi.org/10.1596/978-1-4648-0008-5

Kotler, P., y Lane Keller, K. (2012). Dirección de Marketing (14ed.). México: Pearson Educación. Lalaleo-Analuisa, F.R., Bonilla-Jurado, D.M., y Robles-Salguero, R.E. (2021). Tecnologías de la Información y Comunicación exclusivo para el comportamiento del consumidor desde una perspectiva teórica. Retos Revista de Ciencias de la Administración y Economía, 11(21), pp. 147-164. https://doi.org/10.17163/ret.n21.2021.09.

Lasio, V., Amaya, A., Zambrano, J. y Ordeñana, X. (2020)._Global Entrepreneurship Monitor Ecuador 2019/2020. https://bit.ly/3DtVQIF

Meister, J. C., \& Willyerd, K. (2010). Mentoring millennials. Harvard Business Review

Mentinno. (2020) Ecuador Estado Digital Ene/2020. https://www.formaciongerencial.com/estadodigitalecuador/\#descarga

Moina-Sánchez, P., Morales-Carrasco, L., y Córdova-Pacheco, A. (2020). Crecimiento económico en una región emprendedora en el Ecuador. Retos Revista de Ciencias de la Administración y Economía, 10(19), 65-80. https://doi.org/10.17163/ret.n19.2020.04

Montiel-Méndez O.J., y Soto-Maciel, A. (2020). Un marco exploratorio para el emprendimiento desde una perspectiva evolutiva. Retos Revista de Ciencias de la Administración y Economía, 10(20), pp. 361-373. https://doi.org/10.17163/ret.n20.2020.10

Ortega, I., y Vilanova, N. (2016, 31 de marzo). Generación Z: El ultimo salto generacional. Atrevia y Deusto Business School. https://revistabyte.es/actualidad-it/generacion-z-el-ultimosalto-generacional/

Pico Versoza, L. M. (2017). El emprendimiento por necesidad, una ventana hacia el desarrollo de oportunidades de negocios. INNOVA Research Journal, 2(1), 131-136. https://doi.org/10.33890/innova.v2.n1.2017.133

Pontón, E y Marquez, C. (2016). El marketing como base para el emprendimiento de los millennials. En Palermo Business Review, 65-82

Pulgaron Henao, L. (2019). Factores motivacionales para el trabajo de los diferentes grupos generacionales que componen una empresa manufacturera del oriente antioqueno en el 2019. Universidad de Antioquia 
Schwab, K. (2015, 6 Julio). The Fourth Industrial Revolution: what it means, how to respond. World economic forum. https://www.weforum.org/agenda/2016/01/the-fourth-industrialrevolution-what-it-means-and-how-to-respond/

Senescyt. (2020). Boletín anual Educación Superior, Ciencia, Tecnología e Innovación. https://bit.ly/3sYck6U

Storey, D. J. (1994). New firm growth and bank financing. Small Business Economics, 6(2), 139150. https://doi.org/10.1007/BF01065186

Sumba Nacipucha, N. A., Cueva Estrada, J. M., y López Chila, R. D. (2018). Perspectivas futuras del marketing. Análisis y posibilidades para el mercado guayaquileño. INNOVA Research Journal, 3(7), 71-83. https://doi.org/10.33890/innova.v3.n7.2018.582

United States Census Bureau (2016). World Population by age and sex 2016. https://www.census.gov/population/international/data/worldpop/tool_population.php

Valencia-Arias, J. A. y Marulanda-Valencia, F. A. (2019). Evolución y tendencias investigativas en autoeficacia emprendedora: un análisis bibliométrico. Estudios Gerenciales, 35(151), 219-232. https://doi.org/10.18046/j.estger.2019.151.3277 